\title{
The Association of Fear-Avoidance Beliefs and Self-Reported Knee Function in Patients With a Knee Injury: A Critically Appraised Topic
}

\author{
Francesca Genoese, LAT, ATC, Shelby Baez, MS, LAT, ATC, and Johanna M. Hoch, PhD, LAT, ATC \\ University of Kentucky
}

\begin{abstract}
Clinical Question: Are fear-avoidance beliefs associated with self-reported knee function in patients with a knee injury? Clinical Bottom Line: There is currently consistent, good-quality, patient-oriented evidence that demonstrates an association between fear-avoidance beliefs and self-reported knee function in patients with a knee injury. Future research should longitudinally examine the association of fear-avoidance beliefs and self-reported knee function in patients with a knee injury.
\end{abstract}

Keywords: injury-related fear, patient-reported outcome measures, patient perceptions, patellofemoral pain, anterior cruciate ligament reconstruction

\section{Clinical Scenario}

More than 2.5 million athletic injuries present to emergency departments each year, and approximately 700,000 are knee injuries. ${ }^{1}$ Common knee injuries that may occur during athletic participation include sprains, strains, contusions, fractures, dislocations, and tendinopathies. ${ }^{1,2}$ Among these, two frequently observed knee-related athletic injuries include patellofemoral pain (PFP) and anterior cruciate ligament (ACL) ruptures. ${ }^{3,4}$ After experiencing knee injury, patients encounter physical impairments that are often the primary focus of musculoskeletal rehabilitation. ${ }^{5}$ However, despite the return of adequate objective physical function, return to sport is not always accomplished. ${ }^{6}$ It has been reported that failure to return to sport may be due to biopsychosocial impairments such as deficits in social support, decreases in self-efficacy, or elevated levels of injury-related fear. ${ }^{7}$ Injury-related fear is a specific psychological impairment that may include fear of movement, fear of reinjury, or fear-avoidance beliefs. ${ }^{8}$ Kinesiophobia, which is fear of movement as a result of vulnerability to reinjury, ${ }^{9}$ has been frequently examined as a contextual factor that prevents individuals from returning to sport. ${ }^{6,10}$ Fearavoidance belief, which is a fear of pain that causes avoidance of behaviors that could produce pain or reinjury, is a less frequently examined psychological impairment that may contribute to poor outcomes after sustaining a knee injury. ${ }^{11}$ Other musculoskeletal populations have demonstrated that individuals with elevated levels of fear-avoidance beliefs are likely to adapt a physically inactive lifestyle to avoid a painful experience or reinjury. ${ }^{11,12}$ It has also been previously demonstrated that patients post ACL rupture and patients with PFP report increased fear-avoidance beliefs in addition to deficits in self-reported knee function. ${ }^{13,14}$

Francesca Genoese is a master's student in the Post-Professional Athletic Training Program at the University of Kentucky, Lexington, KY. Shelby Baez is a doctoral research fellow in the Rehabilitation Sciences Department at the University of Kentucky, Lexington, KY. Johanna M. Hoch is an assistant professor in the Division of Athletic Training, and program director of the Professional Masters in Athletic Training Program at the University of Kentucky, Lexington, KY. Abbey Thomas Fenwick, PhD, MEd, University of North Carolina at Charlotte, is the report editor for this article. Address author correspondence to Francesca Genoese at fmgenoese@uky.edu.
Research suggests that there may be an association between psychological impairments and subjective appraisal of knee function..$^{5}$ There is moderate evidence to support that kinesiophobia is associated with self-perceived levels of knee function in patients after ACL rupture. ${ }^{15}$ However, limited literature has explored whether fear-avoidance beliefs are associated with an individual's self-reported knee function in patients with a knee injury, and, to our knowledge, these findings have not been summarized. Therefore, the purpose of this Critically Appraised Topic (CAT) is to synthesize and critically appraise the available evidence that examines the relationship between fear-avoidance beliefs and self-reported knee function in patients with a knee injury.

\section{Focused Clinical Question}

Are fear-avoidance beliefs associated with self-reported knee function in patients with a knee injury?

\section{Search Strategy}

A computerized search was completed in December 2017 (Figure 1). The search terms used were:

- Patient/Client Group: Physically active individuals with a knee injury

- Intervention/Assessment: Fear-avoidance beliefs and knee function

- Comparison: Not applicable

- Outcomes: Fear-Avoidance Beliefs Questionnaire or FABQ, Fear-Avoidance Beliefs Questionnaire for Physical Activity or FABQ-PA, Knee Outcome Survey or KOS, Knee Outcome Survey for Activities of Daily Living or KOS-ADL, Knee Outcome Survey for Sports Activity Scale or KOS-SAS, International Knee Documentation Committee or IKDC, and Knee Injury and Osteoarthritis Outcome Score or KOOS

The sources of evidence searched included:

- EBSCOhost

- CINAHL 


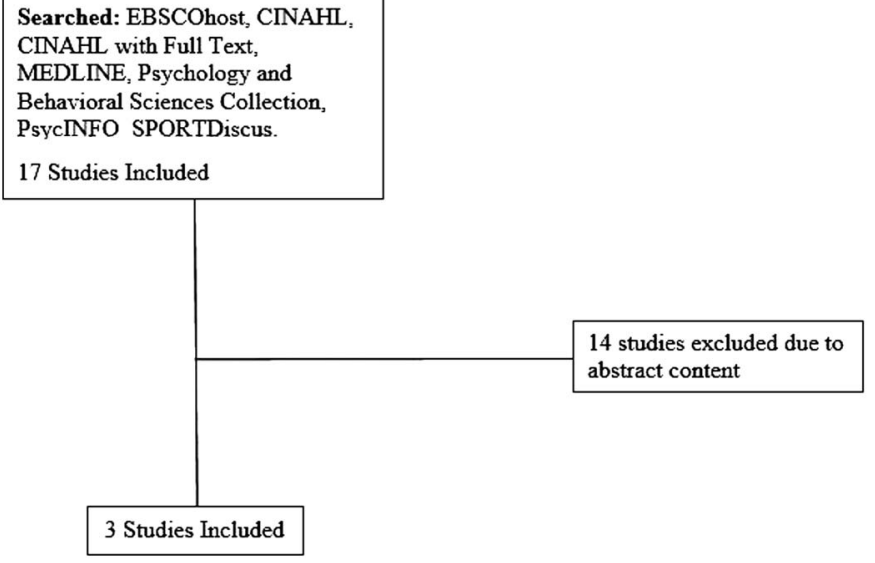

Figure 1 - Summary of search history and included studies.

- CINAHL with Full Text

- MEDLINE

- Psychology and Behavioral Sciences Collection

- PsycINFO

- SPORTDiscus

The criteria for study inclusion were as follows:

- Studies classified as level 3 evidence or higher

- Studies that included participants with a current knee injury or history of knee injury

- Studies that assessed self-reported knee function with use of a patient-reported outcome measure

- Studies that assessed fear-avoidance beliefs as measured by the FABQ

- Studies published in the English language

- Studies published within the last 10 years

The criteria for study exclusion were as follows:

- Studies that examined musculoskeletal conditions unrelated to the knee (e.g., low back pain, additional lower extremity pain conditions not clearly described)

- Studies that examined knee injury or pathology in an arthritic population (e.g., knee osteoarthritis)

- Studies that only included objective measures of knee function

\section{Evidence Quality Assessment}

The STrengthening the Reporting of OBservational studies in Epidemiology (STROBE) Statement was used to determine the validity of the included evidence. Two authors (FG, SB) independently reviewed and scored each study. After independent review, the two authors (FG, SB) reviewed the completed appraisals and reached an agreement about study quality.

\section{Results of Search}

\section{Summary of Search, Best Evidence Appraised, and Key Findings}

- The literature search yielded 17 studies (Figure 1). After review of the abstracts, 14 studies were excluded as they did not meet the inclusion criteria for this CAT. Two cross-sectional studies $^{16,17}$ and one case-control study ${ }^{18}$ met inclusion criteria for this CAT. Study characteristics are categorized in Table 1.

- The three studies assessed the association between fearavoidance beliefs and self-reported knee function in patients with a knee injury. ${ }^{16-18}$ Two of the studies examined the association in PFP patients, ${ }^{17,18}$ while the other study examined this relationship in patients post ACL reconstruction. ${ }^{16}$ All studies used the FABQ or one of the subscales, FABQWork (FABQ-W) or FABQ-PA, to evaluate fear-avoidance beliefs in their respective populations. Self-perceived levels of knee function were measured by the KOS, ${ }^{16} \mathrm{KOS}-\mathrm{ADL},{ }^{17}$ KOS-SAS, ${ }^{16}$ or Anterior Knee Pain Scale (AKPS). ${ }^{18}$

- The results of Ross ${ }^{16}$ demonstrated that fear-avoidance beliefs explained $12 \%$ of the variance in self-perceived functional outcomes in both activities of daily living and sports in patients post ACLR. Piva et al. ${ }^{17}$ and Glaviano et al. ${ }^{18}$ identified a significant negative association between self-reported knee function and fear-avoidance beliefs in patients with patellofemoral pain.

\section{Results of Evidence Quality Assessment}

STROBE items 17 and 22 were excluded from the checklist due to lack of applicability for the studies included in this CAT. Therefore, the maximum score on the STROBE that each study could receive was 20 (Table 1). Glaviano et al. ${ }^{18}$ did not describe any efforts to address potential biases, failed to give adequate description of the setting, locations, and relevant dates, and did not indicate the number of participants with missing data for each variable of interest. Neither Ross ${ }^{16}$ nor Piva et al. ${ }^{17}$ provided a sample size calculation or described any efforts to address potential bias. In addition, both Ross ${ }^{16}$ and Piva et al. ${ }^{17}$ failed to define methods used to examine subgroups and interactions, explain how missing data were addressed, describe any methods that took into account sampling strategy, or indicate the number of participants with missing data for the given variables of interest. Lastly, Ross ${ }^{16}$ failed to provide exclusion criteria for participants in the study.

\section{Clinical Bottom Line}

There is currently consistent, good-quality, patient-oriented evidence that demonstrates an association between fear-avoidance beliefs and self-reported knee function in patients with a knee injury. Future research should longitudinally examine the association of fear-avoidance beliefs and self-reported knee function in patients with a knee injury. Due to the consistent, good-quality evidence, as well as the appropriateness of study designs used to answer the question of this CAT, the grade of A is recommended by the Strength of Recommendation Taxonomy. ${ }^{19}$

\section{Implications for Practice, Education, and Future Research}

The results of this CAT revealed consistent, good-quality, patientoriented evidence that demonstrates the association between fear-avoidance beliefs and self-reported knee function in patients with a knee injury. Ross ${ }^{16}$ investigated the relationship between selfreported knee function and fear-avoidance beliefs after ACL rupture. Forty-eight participants who met the inclusion criteria completed the 


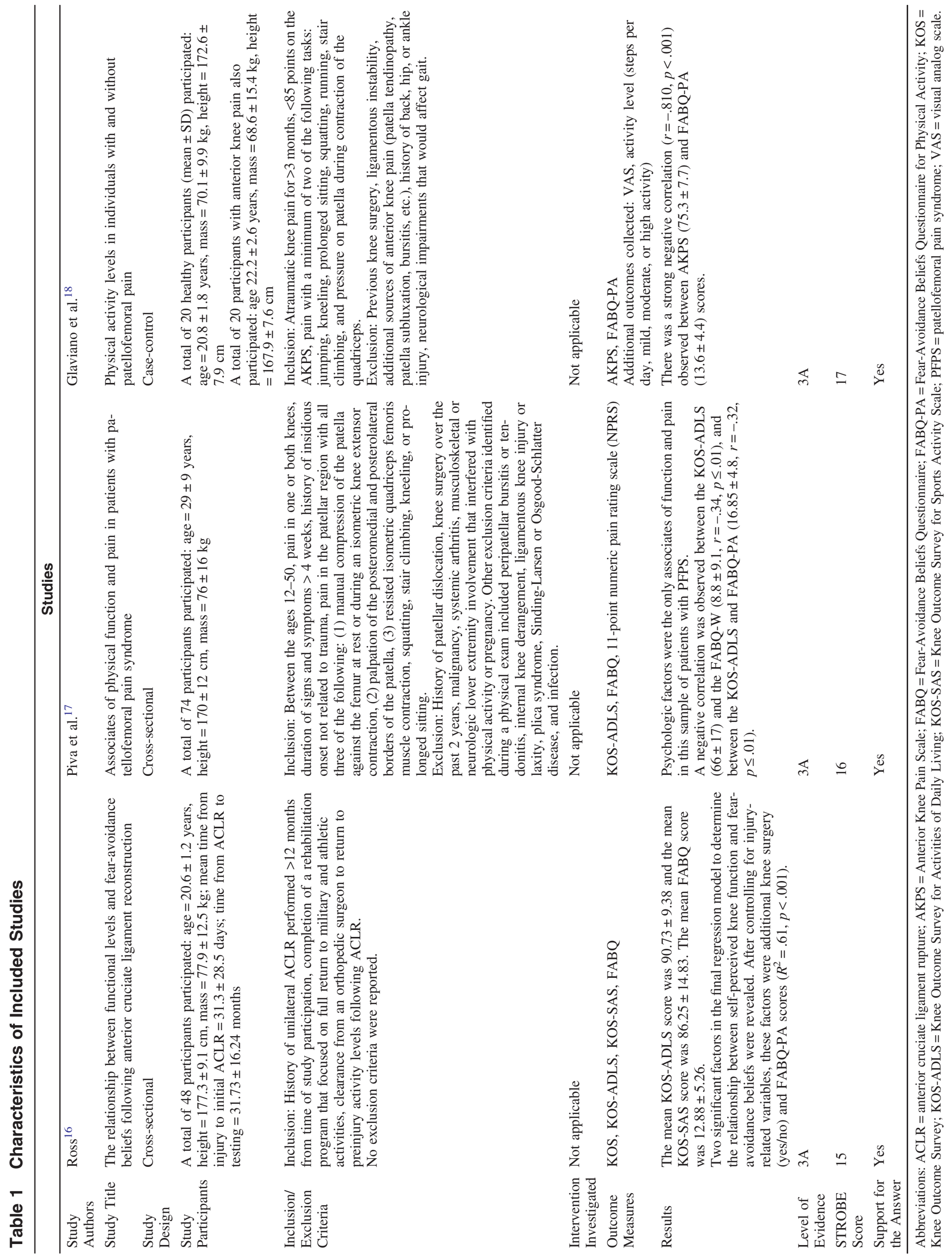


FABQ, KOS-ADLS, and the KOS-SAS before completing KT1000 testing, isokinetic quadriceps testing, and a single-leg hop test to measure for physical impairment. A hierarchical linear regression analysis was used to assess the relationship between self-reported knee function and fear-avoidance beliefs after controlling for injuryrelated variables. Ross ${ }^{16}$ demonstrated that scores on the physical activity subscale of the FABQ significantly contributed to $12 \%$ of the variance in self-perceived functional performance as compared to physical impairment, which only contributed to $1 \%$ of the variance in self-perceived knee function. The final regression model, which included injury-related variables, physical impairment measures, and scores on the FABQ-PA accounted for $61 \%$ of the variance in self-reported knee function scores.

Piva et al. ${ }^{17}$ examined impaired muscle strength, soft tissue length, movement control, postural/biomechanic alterations, pain, and psychological factors and their relationship with physical function, as measured by the KOS-ADLS. Seventy-four participants who were diagnosed with PFP and met the inclusion criteria completed the FABQ-W, FABQ-PA and the Beck Anxiety Index. Measurements of physical impairment, tissue length, foot pronation, Q-angle, tibial torsion, femoral anteversion, and quality movement were also assessed. A forward regression analysis was performed to analyze the relationship between physical impairment and psychological impairment on self-reported knee function. Interestingly, there was no association between physical impairment and self-reported knee function. However, there was a relationship between self-reported function, anxiety, and fearavoidance beliefs. The Beck Anxiety Index, FABQ-W, and FABQ-PA contributed to $18 \%, 6 \%$, and $5 \%$ of the variance in self-perceived levels of knee function, respectively.

Glaviano et al. ${ }^{18}$ utilized a case-control design to examine physical activity levels between individuals with and without PFP. Twenty individuals diagnosed with PFP and who met the inclusion criteria were matched with 20 healthy controls. Participants completed the AKPS and FABQ, and pain was assessed with a VAS. All participants were given a FitBit Charge HR (FitBit Inc., San Francisco, CA) and were instructed to wear the monitor at all times for a 2-week period. Data were retrieved twice per week for steps per day and mild, moderate, and high levels of active minutes. It was demonstrated that individuals diagnosed with PFP took approximately 3,413 fewer steps per day compared to the control group and also reported a reduced number of active minutes per day. Additionally, the researchers discovered a strong negative correlation between fear-avoidance beliefs and selfreported knee function in this study population. Increased levels of fear-avoidance beliefs were associated with decreased levels of self-perceived knee function $(r=.810)$.

Despite consistent results between the studies, differences in the amount of association between fear-avoidance beliefs and selfreported knee function were observed. Participant demographics and eligibility criteria could have influenced the observed association between these variables in each respective study. Ross ${ }^{16}$ included participants post ACLR who were enrolled in a military academy, Glaviano et al. ${ }^{18}$ enrolled general participants with PFP from the collegiate setting between the ages of 18-35, and Piva et al. ${ }^{17}$ recruited participants with PFP between the ages of 12 and 50. Differences in age and in knee pathology could influence the strength and presence of fear-avoidance beliefs. Glaviano et al. ${ }^{18}$ and Ross $^{16}$ may have had a stronger association between fear-avoidance beliefs and self-perceived levels of knee function because their participants were a younger study sample that may be more frequently exposed to fear-eliciting functional situations. Additionally, it has been demonstrated that priorities for physical activity may change with age. ${ }^{20}$ Patients who are older may have less fearavoidance beliefs about physical activity because their priorities may have shifted from regular physical activity to other important factors, for instance, providing for their family. ${ }^{13}$

Furthermore, those patients who engage in a lengthy rehabilitation process, such as a 9-12-month ACL rupture rehabilitation program, may experience increased fear-avoidance beliefs because of the rigorous rehabilitation process and time loss associated with an ACL injury. ${ }^{6}$ These factors could help to explain the differences in association between fear-avoidance beliefs and self-reported knee function as examined by Ross, ${ }^{16}$ Piva et al., ${ }^{17}$ and Glaviano et al. ${ }^{18}$

The studies reviewed all utilized the FABQ, a dimensionspecific patient-reported outcome (PRO), designed to measure fearavoidance beliefs about work (FABQ-W) and physical activity (FABQ-PA). ${ }^{14}$ The FABQ was originally created to examine patients' beliefs about how low back pain affected work and physical activity. ${ }^{15}$ The FABQ contains a total of 16 items, with 11 items in the FABQ-W subscale, and 5 items in the FABQ-PA subscale. Each item is scored on a Likert scale from 0 to 6 , where lower scores represent lower levels of fear-avoidance beliefs and higher scores represent elevated levels of fear-avoidance beliefs. In the examined studies, Glaviano et al. ${ }^{18}$ utilized the original FABQPA, while Ross ${ }^{16}$ and Piva et al. ${ }^{17}$ used the FABQ-W and a modified version of the FABQ-PA specific for patients with a knee pathology to evaluate fear-avoidance beliefs.

Despite observed associations between fear-avoidance beliefs and self-reported levels of knee function, the results of these studies must be interpreted with caution. The FABQ was designed to examine fear-avoidance beliefs in patients with low back pain, and modified versions of the FABQ, as discussed previously, have been developed for other musculoskeletal populations. However, limited research has examined the psychometric properties of these modified versions of the FABQ. Future research should examine the psychometric properties, including reliability, validity, consistency, responsiveness, and cut-off scores for the modified versions of the FABQ. The assessment of these properties could enhance implementation of psychologicallyinformed clinical practice by rehabilitation specialists.

Additionally, studies that have examined kinesiophobia in patients after ACLR have reported a decrease in fear during the rehabilitation process as well as a resurgence of fear upon return to sport that negatively impacts self-perceived function. ${ }^{6}$ Future research should explore fear-avoidance beliefs longitudinally in patients with knee injuries, specifically throughout the postoperative and return-to-sport phase. Longitudinal data on the relationship between fear-avoidance beliefs and self-reported knee function in these patients could provide further insight into the cycle of biopsychosocial impairments that accompany initial injury, postoperative rehabilitation, and return to sports participation. Moreover, implementation of the FABQ at different time points in the injury and recovery process could help to guide clinicians on appropriate and patient-centered psychosocial intervention strategies to better help patients manage their fears and return to physical activity.

Future studies should also examine the effects of psychosocial intervention strategies on fear-avoidance beliefs. Cognitive behavioral interventions that have been used to decrease fear in other musculoskeletal populations include imagery, self-talk, cognitive restructuring, graded exposure, and progressive muscle relaxation. ${ }^{10}$ Additionally, there is sufficient evidence to support the use of mindfulness-based interventions for increasing mindfulness, managing negative emotions and perceived stress, and improving overall well-being. ${ }^{21}$ These intervention strategies may be useful for patients 
with a knee injury who have elevated fear-avoidance beliefs because these strategies can help patients anticipate their pain, recognize and change negative thoughts, and could reduce tension and anxiety. ${ }^{10}$ Such intervention strategies, as well as collaboration with behavioral health specialists, could improve rehabilitation, recovery, activities of daily living, sport participation, and quality of life for this population.

After experiencing a knee injury, individuals are likely to encounter physical impairments, but may also experience biopsychosocial impairments. Biopsychosocial impairments, specifically fearavoidance beliefs, may impede self-reported knee function, amongst other outcomes, including long-term physical activity engagement. This CAT has summarized the evidence to support an association between fear-avoidance beliefs and self-reported knee function in patients with a knee injury. Use of dimension-specific PROs, such as the FABQ, could allow clinicians to assess these biopsychosocial impairments and to evaluate the efficacy of a patient-centered psychosocial intervention to decrease fear in patients with knee injury. Psychologically-informed clinical practice could further aid in the administration of patient-centered, holistic healthcare for this population. Acknowledgment and treatment of fear-avoidance beliefs in patients with a knee injury are imperative to help patients reduce fearavoidance behaviors and increase self-reported levels of knee function. This CAT should be reviewed in 2 years (2020) to determine whether additional evidence is present that can provide further insight on the association between fear-avoidance beliefs and self-perceived levels of knee function in patients with a knee pathology.

\section{CAT Kill Date: September 2020}

CATs have limited life and should be revisited approximately 2 years after publication (see https://doi.org/10.1123/ijatt. 2018-0093).

\section{References}

1. Gage BE, McIlvain NM, Collins CL, Fields SK, Dawn Comstock R. Epidemiology of 6.6 million knee injuries presenting to United States emergency departments from 1999 through 2008. Acad Emerg Med. 2012;19(4):378-385. PubMed ID: 22506941 doi:10.1111/j.15532712.2012.01315.x

2. Ingram JG, Fields SK, Yard EE, Comstock RD. Epidemiology of knee injuries among boys and girls in US high school athletics. Am J Sports Med. 2008;36(6):1116-1122. PubMed ID: 18375784 doi: 10.1177/0363546508314400

3. Boling M, Padua D, Marshall S, Guskiewicz K, Pyne S, Beutler A. Gender differences in the incidence and prevalence of patellofemoral pain syndrome. Scan J Med Sci Sport. 2010;20(5):725-730. doi:10. 1111/j.1600-0838.2009.00996.x

4. Ardern CL. Anterior cruciate ligament reconstruction-not exactly a one-way ticket back to the preinjury level: a review of contextual factors affecting return to sport after surgery. Sports Health. 2015;7(3): 224-230. PubMed ID: 26131299 doi:10.1177/1941738115578131

5. Prodromos CC, Han Y, Rogowski J, Joyce B, Shi K. A meta-analysis of the incidence of anterior cruciate ligament tears as a function of gender, sport, and a knee injury-reduction regimen. Arthroscopy. 2007;23(12): 1320-1325.e6. PubMed ID: 18063176 doi:10.1016/j.arthro.2007.07.003

6. Chmielewski TL, Jones D, Day T, Tillman SM, Lentz TA, George SZ. The association of pain and fear of movement/reinjury with function during anterior cruciate ligament reconstruction rehabilitation. J Orthop Sports Phys Ther. 2008;38(12):746-753. PubMed ID: 19047767 doi:10.2519/jospt.2008.2887

7. Burland JP, Toonstra J, Werner JL, Mattacola CG, Howell DM, Howard JS. Decision to return to sport after anterior cruciate ligament reconstruction, part I: a qualitative investigation of psychosocial factors. $J$ Athl Train. 2018;53(5):452-463. doi:10.4085/1062-6050-313-16

8. Ardern CL, Taylor NF, Feller JA, Whitehead TS, Webster KE. Psychological responses matter in returning to preinjury level of sport after anterior cruciate ligament reconstruction surgery. Am J Sports Med. 2013;41(7):1549-1558. PubMed ID: 23733635 doi:10. 1177/0363546513489284

9. Kori SH, Miller RP, Todd DD. Kinisophobia: a new view of chronic pain behavior. Pain Manag. 1990;3:35-43.

10. Hsu C-J, Meierbachtol A, George SZ, Chmielewski TL. Fear of reinjury in athletes: implications for rehabilitation. Sports Health. 2017;9(2): 162-167. PubMed ID: 27590793 doi:10.1177/1941738116666813

11. Vlaeyen JW, Linton SJ. Fear-avoidance and its consequences in chronic musculoskeletal pain: a state of the art. Pain. 2000;85(3):317-332. PubMed ID: 10781906 doi:10.1016/S0304-3959(99)00242-0

12. Filbay SR, Crossley KM, Ackerman IN. Activity preferences, lifestyle modifications and re-injury fears influence longer-term quality of life in people with knee symptoms following anterior cruciate ligament reconstruction: a qualitative study. J Physiother. 2016;62(2):103-110. PubMed ID: 26994508 doi:10.1016/j.jphys.2016.02.011

13. Hoch JM, Sinnott CW, Robinson KP, Perkins WO, Hartman JW. The examination of patient-reported outcomes and postural control measures in patients with and without a history of ACL reconstruction: a case control study. J Sport Rehabil. 2018;27(2):170-176. doi: 10.1123/jsr.2016-0105

14. Piva SR, Fitzgerald GK, Wisniewski S, Delitto A. Predictors of pain and function outcome after rehabilitation in patients with patellofemoral pain syndrome. J Rehabil Med. 2009;41(8):604-612. PubMed ID: 19565153 doi:10.2340/16501977-0372

15. Cozzi AL, Dunn KL, Harding JL, McLeod TCV, Bacon CEW. Kinesiophobia after anterior cruciate ligament reconstruction in physically active individuals. J Sports Rehabil. 2015;24(4): 434-439. doi:10.1123/jsr.2014-0196

16. Ross MD. The relationship between functional levels and fearavoidance beliefs following anterior cruciate ligament reconstruction. J Orthop Traumatol. 2010;11(4):237-243. PubMed ID: 21116674 doi:10.1007/s10195-010-0118-7

17. Piva SR, Fitzgerald GK, Irrgang JJ, et al. Associates of physical function and pain in patients with patellofemoral pain syndrome. Arch Phys Med Rehabil. 2009;90(2):285-295. PubMed ID: 19236982 doi: 10.1016/j.apmr.2008.08.214

18. Glaviano NR, Baellow A, Saliba S. Physical activity levels in individuals with and without patellofemoral pain. Phys Ther Sport. 2017;27: 12-16. PubMed ID: 28780340 doi:10.1016/j.ptsp.2017.07.002

19. Ebell MH, Siwek J, Weiss BD, et al. Strength of recommendation taxonomy (SORT): a patient-centered approach to grading evidence in the medical literature. J Am Board Fam Pract. 2004;17(1):59-67. PubMed ID: 15014055 doi:10.3122/jabfm.17.1.59

20. Tjong VK, Murnaghan ML, Nyhof-Young JM, Ogilvie-Harris DJ. A qualitative investigation of the decision to return to sport after anterior cruciate ligament reconstruction: to play or not to play. Am J Sports Med. 2014;42(2):336-342. PubMed ID: 24197615 doi:10.1177/ 0363546513508762

21. Petterson H, Olson BL. Effects of mindfulness-based interventions in high school and college athletes for reducing stress and injury, and improving quality of life. J Sport Rehabil. 2017;26(6):578-587. PubMed ID: 27632857 doi:10.1123/jsr.2016-0047 